\title{
Analysis of Thermo-Magneto-Elastic Nonlinear Dynamic Response of Shallow Conical Shells
}

\author{
Shende Cen, Jiachu Xu \\ School of Mechanics and Construction Engineering, Jinan University, Guangzhou, China \\ Email: 875545520@qq.com,txjch@jnu.edu.cn
}

How to cite this paper: Cen, S.D. and $\mathrm{Xu}$, J.C. (2018) Analysis of Thermo-MagnetoElastic Nonlinear Dynamic Response of Shallow Conical Shells. Engineering, 10, 837-850.

https://doi.org/10.4236/eng.2018.1012058

Received: September 13, 2018

Accepted: December 11, 2018

Published: December 14, 2018

Copyright ( $) 2018$ by authors and Scientific Research Publishing Inc. This work is licensed under the Creative Commons Attribution International License (CC BY 4.0).

http://creativecommons.org/licenses/by/4.0/

(c) (i) Open Access

\begin{abstract}
The dynamic response study on thermo-magneto-elastic behavior of shallow conical shell in a time-dependent magnetic field is investigated, and the dynamic responses of displacement of shallow conical shell under mechanical loads, electromagnetic fields and temperature field coupling are analyzed. Based on Maxwell's equations, heat conduction equation and nonlinear equations of classical plates and shells, the nonlinear dynamic response governing equations are derived. The electromagnetic field and temperature field equations are solved using variable separating technique, the nonlinear elastic field equations are solved by Galerkin method. The variation of temperature, magnetic field intensity and displacement with time under the coupling effect of the applied magnetic field and the surface uniform load were obtained. The influence of frequency of the applied magnetic field on the displacement wave forms is discussed.
\end{abstract}

\section{Keywords}

Thermo-Magneto-Elastic, Shallow Conical Shell, Nonlinear Dynamic Response, Galerkin Method

\section{Introduction}

Thermo-magneto-elasticity is a new subject to study the strength, stiffness and stability of elastic components under the combined action of electromagnetic, temperature and deformation. Magneto-elasticity is the theory of studying the coupling between electromagnetic and deformation, thermo-elasticity combines elasticity and heat conduction to study the coupling theory between temperature field and elastic field. The thermo-magneto-elasticity includes heat conduction theory, classical elasticity theory and electromagnetic theory. These theories are applied to solve the coupling problems of temperature field, electromagnetic 
field and elastic field of conductive elastic elements located in magnetic field and considering thermal effect.

With the wide application of electromagnetic structure in the high-tech field, many components work in the environment of temperature change in engineering, the research on the thermos-elastic phenomenon of electromagnetic coupling has a strong engineering background and theoretical value [1] [2]. The elastic elements and structures in high energy-varying magnetic field under mechanical loads can produce various stresses. In addition to mechanical stress, there are the thermal stress generated by the induced eddy current losses, and the magnetic stress generated by the Lorentz force. These stresses affect each other, and to be high nonlinear. Previous studies on the thermo-magneto-elastic problem of plate and shell were mainly based on the simplified theory and the linear theories. However, in actual situation, most plate and shell structure are in the temperature-varying high-energy electromagnetic fields, which is a highlycoupled nonlinearity.

The key to the thermo-magneto-elastic analysis is the solution of the thermo-magneto-elastic equation. Xing studied on the dynamic response and quasi-static response of the rectangular plate thermo-magneto-elastic in the temperature-varying magnetic field with difference method. The curves of temperature, magnetic field intensity, induced current, and thermal elastic stress and displacement with time are obtained [3]. Higuchi et al. studied the cylinder affected by the change magnetic field and the thermo-magneto-elastic stress of the hollow cylindrical shell with separation of variables [4] [5]. Wang Ping studied the chaotic motion of the large deflection simple support plate under the coupling effect of mechanical loads, electromagnetic and temperature field [6] [7]. Alberto Milazzo presented a new one-dimensional model for the dynamic problem of magneto-electro-elastic generally laminated beams [8]. Based on the successful validation of the model, new results for free vibrations of functionally graded magneto-electro-elastic beams are presented. Zhang Lang analyzed the buckling and vibration of functionally graded magneto-electro-thermo-elastic circular cylindrical shells. Kattimani studied the Control of geometrically nonlinear vibrations of functionally graded magneto-electro-elastic plates [9] [10].

It is very difficult to study the nonlinear dynamic response of a shallow conical shell in an alternating magnetic field and subjected to mechanical loads. The study on the nonlinear dynamic response of the shell is very rare. In this paper, the electromagnetic field equations are derived based on Maxwell's equations and Ohm's law. Based on the heat conduction equation and the heat balance equation, temperature field equations are derived. Based on nonlinear equations of classical plates and shells, considering the coupling effect of the Lorentz force and temperature stress, nonlinear magneto-elastic heat equations of shallow conical shell are deduced. Applying Galerkin method, the solution of thermomagneto-elastic equation, the rule of temperature, magnetic field and displacement varying with time under the coupling effect of the applied magnetic field 
and surface uniform stable mechanical loads are obtained.

\section{Basic Equations}

Considering shallow conical shell with thickness $h$, radium $a$ and pyramid dip $\varphi$, whose neuter plane is showed in Figure 1 Assume that shallow conical shell in alternating magnetic field works under axisymmetric state, whose outer surface is subjected to normal stable mechanical load. The orthogonal curvilinear coordinate $(r, \theta, z)$ is established in Figure 1, where $r, \theta, z$ are radium, annular and normal coordinate of shallow conical shell respectively. The applied magnetic field intensity is $H\left(H_{r}, 0,0\right)$ and Mechanical load is $P\left(0,0, P_{z}\right) . H_{r}$ is a function of the coordinate $z$ and time $t$, and $P_{z}$ is constant.

\subsection{Electro Dynamics Equations}

In the absence of lateral current and the influence of displacement current and volume charge density is not considered, according to Maxwell equation and generalized Ohm's law, the electrodynamics equations are:

$$
\begin{aligned}
& \operatorname{rot} E=-\frac{\partial B}{\partial t} \\
& \operatorname{rot} H=J \\
& J=\sigma(E-V B) \\
& B=\mu H
\end{aligned}
$$

where $E$ is electric field intensity, $B$ is magnetic induction intensity, $H$ is magnetic field intensity, $J$ is current density, $V$ is velocity, $\sigma$ is admittance, $\mu$ is permeability, rot is rotation.

Ignoring the mechanical electric effect and considering the axial symmetry, the electrodynamics Equation (1) of shallow conical shell can be simplified as

$$
\begin{aligned}
& \frac{\partial H_{r}}{\partial t}=\frac{\phi^{2}}{\mu \sigma} \frac{\partial^{2} H_{r}}{\partial z^{2}} \\
& J_{\theta}=\phi \frac{\partial H_{r}}{\partial z}
\end{aligned}
$$

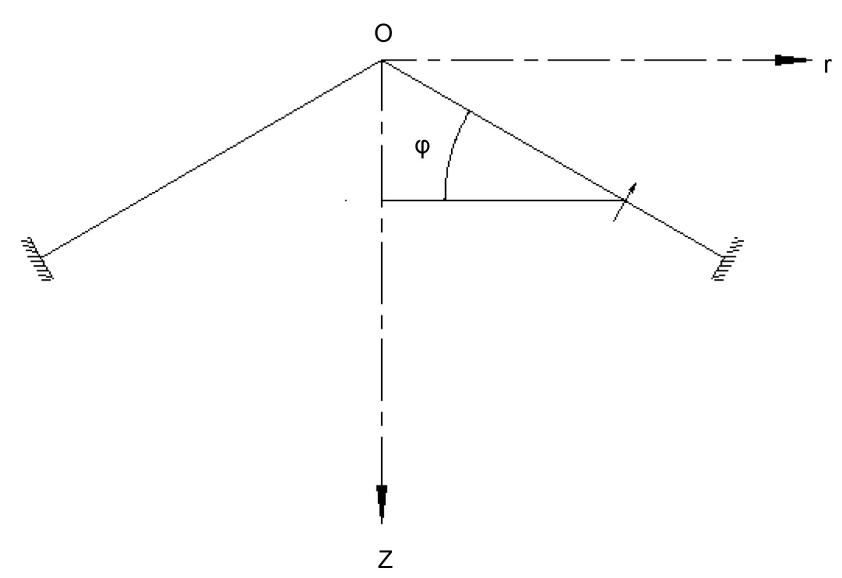

Figure 1. The diagram of shallow conical shell. 


\subsection{Temperature Field}

As electromagnetic field varying with time induces current in shallow conical shell, which formats Joule heating effect, that is induction current loss. As to shallow conical shell, it can approximately assume that current distribute uniformly in shallow conical shell because of a low frequency of the applied magnetic field and current, then the current loss per unit time per volume can be calculated by the following formula:

$$
Q=\frac{J_{\theta}^{2}}{\sigma}
$$

Set the initial temperature of shallow conical shell to zero, it is heated by Joule heating effect from $t=0$, heat exchange exist among the Inner and outer surface and the bottom of the shell and media whose external temperature is zero. According to Fourier heat transfer law and energy conservation law, the control equation of heat conduction is established, which means that the transient temperature field $T(z, t)$ of shallow conical shell should satisfy the followed equation:

$$
\frac{\partial T}{\partial t}=k \nabla^{2} T+\frac{Q}{\rho c}
$$

where $\rho$ is material mass density, $c$ is specific heat capacity, $k$ is coefficient of thermal conductivity, $\nabla^{2}$ is Laplace operator.

In the axial symmetry condition, the control equation of heat conduction of shallow conical shell can be simplified to

$$
\frac{\partial T}{\partial t}=k \varphi^{2} \frac{\partial^{2} T}{\partial z^{2}}+\frac{Q}{\rho c}
$$

Based on heat exchange law and the condition that the external temperature of media is zero, the heat balance equation of the current-carrying shell's internal and external surface can be established. Thus, the boundary condition is

$$
\pm \frac{\partial T}{\partial z}+\Lambda T=0, \text { at } z= \pm \frac{h}{2}
$$

where, $\Lambda$ is thermal coefficient.

\subsection{Elastic Field}

The shallow conical shell in the time dependent electromagnetic field also suffer the temperature stress induced by Joule heat and Lorentz force in addition to the external mechanical loads $P$, The Lorentz force can be expressed as

$$
f_{z}=-\mu H_{r} \frac{\partial H_{r}}{\partial z}
$$

where $f_{z}$ is $z$ direction component of Lorentz force $f$.

When discussing the thermo-magneto-elastic nonlinear problem of a shallow conical shell, the Kichhoff-love straight normal hypothesis is adopted, that the normal section perpendicular to the neutral surface before deformation of the 
shell remains a straight line after deformation and perpendicular to the neutral plane after deformation, and its length remains unchanged. Based on this assumption, the radial, toroidal and normal displacements of any point in a shell with a distance of $z$ from the neutral plane under axial symmetry conditions can be expressed as:

$$
\begin{aligned}
& u(z)=u-z \frac{\partial w}{\partial r} \\
& v(z)=0 \\
& w(z)=w
\end{aligned}
$$

Among them, $u$ and $w$ are the radial displacement and deflection at any point on the neutral surface of the shell respectively.

Considering the Von-Karman type large deflection geometric relation of conical shell, the strain-displacement relation at any point in the shell is obtained:

$$
\begin{aligned}
& \varepsilon_{r}(z)=\frac{\partial u}{\partial r}+\varphi \frac{\partial w}{\partial r}+\frac{1}{2}\left(\frac{\partial w}{\partial r}\right)^{2}-z \frac{\partial^{2} w}{\partial r^{2}} \\
& \varepsilon_{\theta}(z)=\frac{u}{r}-z \frac{1}{r} \frac{\partial w}{\partial r}
\end{aligned}
$$

The physical equation of shallow conical shell considering temperature change can be calculated according to follow formula.

$$
\begin{aligned}
& \sigma_{r}=\frac{E}{1-v^{2}}\left[\left(\varepsilon_{r}+v \varepsilon_{\theta}\right)-(1+v) \alpha T\right] \\
& \sigma_{\theta}=\frac{E}{1-v^{2}}\left[\left(\varepsilon_{\theta}+v \varepsilon_{r}\right)-(1+v) \alpha T\right]
\end{aligned}
$$

The $E, v, \alpha$ in the formula represents the elastic modulus, Poisson's ratio and linear thermal expansion coefficient respectively, $\sigma_{r}, \sigma_{\theta}$ are $r, \theta$ stress respectively.

In the axial symmetry condition, considering the coupling effect of Lorentz force, temperature stress and mechanical load, according to the classical theory of plates and shells, the control equation of shallow conical shell can be derived as follows:

$r^{2} \frac{\partial^{2} u}{\partial r^{2}}+r \frac{\partial u}{\partial r}-u+(1-v) r \varphi \frac{\partial w}{\partial r}+\frac{(1-v)}{2} r\left(\frac{\partial w}{\partial r}\right)^{2}+r^{2}\left(\varphi+\frac{\partial w}{\partial r}\right) \frac{\partial^{2} w}{\partial r^{2}}=0$

$D\left(r \frac{\partial^{3} w}{\partial r^{3}}+\frac{\partial^{2} w}{\partial r^{2}}-\frac{1}{r} \frac{\partial w}{\partial r}\right)-r\left\{A\left[\frac{\partial u}{\partial r}+\varphi \frac{\partial w}{\partial r}+\frac{1}{2}\left(\frac{\partial w}{\partial r}\right)^{2}+v \frac{u}{r}\right]-N^{T}\right\}\left(\varphi+\frac{\partial w}{\partial r}\right)$

$-\frac{1}{2}\left(P_{z}+F_{z}\right) r^{2}=\rho h \int_{0}^{r} r \frac{\partial^{2} w}{\partial t^{2}} \mathrm{~d} r$

where $A$ and $D$ are extension rigidity and bending rigidity of shallow conical shell respectively. $F_{z}$ and $N^{T}$ are the resultant forces of Lorentz force in the transverse direction and the film force produced by thermal stress respectively.

$$
F_{z}=\int_{-h / 2}^{h / 2} f_{z} \mathrm{~d} z, \quad N^{T}=\frac{E \alpha}{1-v} \int_{-h / 2}^{h / 2} T(z) \mathrm{d} z
$$




\subsection{Non-Dimensionalization}

To facilitate the calculation, the following dimensionless parameters are introduced

$$
\begin{aligned}
& \bar{r}=\frac{r}{a}, \bar{H}_{r}=\frac{H_{r}}{H_{0}}, \tau=\frac{4 \varphi^{2} t}{\mu \sigma h^{2}}, \bar{u}=\frac{4 a}{h^{2}} u, \bar{w}=\frac{2 w}{h}, k=\frac{2 a}{h} \varphi, \bar{z}=\frac{2 z}{h}, \\
& \bar{J}_{\theta}=\frac{h J_{\theta}}{2 H_{0}}, \bar{Q}=\frac{h^{2} \sigma Q}{4 H_{0}^{2} \varphi^{2}}, \bar{T}=\frac{c \rho T}{\mu H_{0}^{2}}, \bar{h}=h \Lambda / 2,\left(\bar{F}_{z}, \bar{P}_{z}\right)=\frac{a^{4}}{D h^{2}}\left(F_{z}, P_{z}\right), \\
& \bar{P}_{z}=\frac{8 a^{4}}{A h^{3}} P_{z}, \bar{N}^{T}=\frac{a^{2}}{D} N^{T}, \bar{f}_{z}=\frac{h}{2 \mu H_{0}^{2}} f_{z},\left(\bar{\sigma}_{r}, \bar{\sigma}_{\theta}\right)=\frac{4 a^{2}\left(1-v^{2}\right)}{h^{2} E}\left(\sigma_{r}, \sigma_{\theta}\right), \\
& \varsigma_{1}=k \sigma \mu, \varsigma_{2}=\frac{A h^{2}}{4 D}, \varsigma_{3}=\frac{32 \rho a^{4} \varphi^{4}}{D \sigma^{4} \mu^{2} h}
\end{aligned}
$$

The non-dimensional forms of Equation (2), (3), (5) and (11) are given as follows

$$
\begin{gathered}
\frac{\partial \bar{H}_{r}}{\partial \tau}=\frac{\partial^{2} \bar{H}_{r}}{\partial \bar{z}^{2}} \\
\bar{Q}=\bar{J}_{\theta}^{2} \\
\frac{\partial \bar{T}}{\partial \tau}=\varsigma_{1} \frac{\partial^{2} \bar{T}}{\partial \bar{z}^{2}}+\bar{Q} \\
\bar{r}^{2} \frac{\partial^{2} \bar{u}}{\partial \bar{r}^{2}}+\bar{r} \frac{\partial \bar{u}}{\partial \bar{r}}-\bar{u}+(1-v) k \bar{r} \frac{\partial \bar{w}}{\partial \bar{r}}+\frac{1}{2}(1-v) \bar{r}\left(\frac{\partial \bar{w}}{\partial \bar{r}}\right)^{2}+\bar{r}^{2}\left(k+\frac{\partial \bar{w}}{\partial \bar{r}}\right) \frac{\partial^{2} \bar{w}}{\partial \bar{r}^{2}}=0 \\
\bar{r} \frac{\partial^{3} \bar{w}}{\partial \bar{r}^{3}}+\frac{\partial^{2} \bar{w}}{\partial \bar{r}^{2}}-\frac{1}{\bar{r}} \frac{\partial \bar{w}}{\partial \bar{r}}-\varsigma_{2} \bar{r}\left\{\left[\frac{\partial \bar{u}}{\partial \bar{r}}+k \frac{\partial \bar{w}}{\partial \bar{r}}+\frac{1}{2}\left(\frac{\partial \bar{w}}{\partial \bar{r}}\right)^{2}+v \frac{\bar{u}}{\bar{r}}\right]-N^{T}\right\}\left(k+\frac{\partial \bar{w}}{\partial \bar{r}}\right) \\
-\left(\bar{P}_{z}+\bar{F}_{z}\right) \bar{r}^{2}=\varsigma_{3} \int_{0}^{\bar{r}} \bar{r} \frac{\partial^{2} \bar{w}}{\partial \tau^{2}} d \bar{r}
\end{gathered}
$$

In Equation (17), the expressions of $\bar{F}_{z}$ and $N^{T}$ are respectively as follow:

$$
\bar{F}_{z}=\int_{-1}^{1} \bar{f}_{z} \mathrm{~d} \bar{z}, \quad N^{T}=\int_{-1}^{1} \bar{T}(\bar{z}, \tau) \mathrm{d} \bar{z}
$$

Consider the following boundary conditions and initial conditions of electromagnetic field, temperature field and elastic field

$$
\begin{array}{cc}
\bar{H}_{r}=\varphi(\tau) & \text { at } \bar{z}=1 \\
\bar{H}_{r}=0 & \text { at } \bar{z}=-1 \\
\bar{H}_{r}=0 & \text { when } \tau=0 \\
\pm \frac{\partial \bar{T}}{\partial \bar{z}}+\bar{h} \bar{T}=0 \quad \text { at } \bar{z}= \pm 1 \\
\bar{T}(\bar{z}, \tau)=0 \quad \text { when } \tau=0 \\
\bar{w}=0, \quad \frac{\partial \bar{w}}{\partial \bar{r}}=0, \quad \bar{u}=0 \text { at } \bar{r}=1 \\
\frac{\partial \bar{w}}{\partial \bar{r}}=0, \bar{u} \text { is finite at } \bar{r}=0
\end{array}
$$




$$
\bar{w}=\frac{\partial \bar{w}}{\partial \tau}=0 \text { and } \bar{u}=\frac{\partial \bar{u}}{\partial \tau}=0 \text { when } \tau=0
$$

\section{Solution}

To solve the governing Equation (17), the first step is to solve the electromagnetic field Equation (14) to obtain the magnetic field strength $\bar{H}_{r}$ and Lorentz force $\bar{f}_{z}$. Solving Equation (16) gets the temperature field distribution $\bar{T}$, and obtains $\bar{F}_{z}$ and $N^{T}$ according to formula (18).

\subsection{Electromagnetic Field}

Equation (14) is solved by separation of variables. In order to make non-homogeneous boundary Condition (19) homogeneous, new unknown function $h_{r}(\bar{z}, \tau)$ is introduced

$$
h_{r}(\bar{z}, \tau)=\bar{H}(\bar{z}, \tau)-\frac{\bar{z}+1}{2} \phi(\tau)
$$

Substituting Formula (24) in to Equation (14), boundary conditions and initial Condition (19) can be written as follows

$$
\begin{gathered}
\frac{\partial h_{r}}{\partial \bar{z}^{2}}=\frac{\partial h_{r}}{\partial \tau}+\frac{1+\bar{z}}{2} \frac{\mathrm{d} \phi(\tau)}{\mathrm{d} \tau} \\
h_{r}=0 \text { at } \bar{z}= \pm 1 \\
h_{r}(\bar{z}, 0)=-\phi(0) \frac{1+\bar{z}}{2} \text { when } \tau=0
\end{gathered}
$$

Assume that the solution of Equation (25) satisfy the Conditions (26) and (27) is following series form

$$
h_{r}=\sum_{n=1}^{\infty} \psi_{n}(\tau) \cos \left(k_{n} \bar{z}\right)
$$

where $\psi(\tau)$ is the function of $\tau, k_{n}$ is the position root of function $\cos \left(k_{n}\right)=0$, that is

$$
k_{n}=\frac{2 n-1}{2} \pi \quad(n=1,2,3, \cdots)
$$

Substituting Formula (28) in the Equation (25), both sides of Equation (25) multiply $\cos \left(k_{m} \bar{z}\right)$, using the orthogonality of trigonometric function, it obtains

$$
\frac{\mathrm{d} \psi(\tau)}{\mathrm{d} \tau}+k_{n}^{2} \psi(\tau)+\int_{-1}^{1} \frac{\mathrm{d} \phi(\tau)}{\mathrm{d} \tau} \frac{1+\bar{z}}{2} \cos \left(k_{n} \bar{z}\right) \mathrm{d} \bar{z}=0
$$

With initial Condition (22), the solution of Equation (30) is

$$
\psi_{n}=\frac{(-1)^{n}}{k_{n}} \bar{\psi}_{n}(\tau)
$$

where $\bar{\psi}(\tau)=\int_{0}^{\tau} \mathrm{e}^{-k_{n}^{2}(\tau-x)} \frac{\mathrm{d} \varphi(x)}{\mathrm{d} x} \mathrm{~d} x$

Thus, the expressions of non-dimensional magnetic field intensity $\bar{H}_{r}$, in- 
duced current and Lorentz force respectively are

$$
\begin{aligned}
& \bar{H}_{r}=\sum_{n=1}^{\infty} \frac{(-1)^{n}}{k_{n}} \bar{\psi}(\tau) \cos \left(k_{n} \bar{z}\right)+\frac{1+\bar{z}}{2} \varphi(\tau) \\
& \bar{J}_{\theta}=\sum_{n=1}^{\infty}(-1)^{n+1} \sin \left(k_{n} \bar{z}\right) \bar{\psi}(\tau)+\frac{1}{2} \varphi(\tau) \\
& \bar{f}_{z}=\left[\sum_{m=1}^{\infty}(-1)^{m} \bar{\psi}_{m}(\tau) \sin \left(k_{m} \bar{z}\right)-\frac{1}{2} \varphi(\tau)\right]\left[\sum_{n=1}^{\infty} \frac{(-1)^{n}}{k_{n}} \bar{\psi}(\tau) \cos \left(k_{n} \bar{z}\right)+\frac{1+\bar{z}}{2} \varphi(\tau)\right]
\end{aligned}
$$

\subsection{Temperature Field}

Based on the separation of variables, assume that the solution of Equation (16) which satisfies the boundary Condition (20) shows the following form

$$
\bar{T}=\sum_{i=1}^{\infty} b_{i}(\tau) \cos \left(a_{i} \bar{z}\right)
$$

where $a_{i}$ is confirmed by $\tan a_{i}=\frac{\bar{h}}{a_{i}}$. Both sides of Equation (12) multiply $\cos \left(a_{j} \bar{z}\right)$, using the orthogonality of trigonometric function, it obtains

$$
\frac{\partial b_{i}(\tau)}{\partial \tau}+\varsigma_{1} a_{i}^{2} b_{i}(\tau)-\frac{\bar{h}^{2}+a_{i}^{2}}{\bar{h}+\bar{h}^{2}+a_{i}^{2}} \int_{-1}^{1} \bar{Q}(\bar{z}, \tau) \cos \left(a_{i} \bar{z}\right) \mathrm{d} \bar{z}=0
$$

The solution of Equation (34) is

$$
b_{i}(\tau)=\frac{\bar{h}^{2}+a_{i}^{2}}{\bar{h}+\bar{h}^{2}+a_{i}^{2}}\left[\sum_{m=1}^{\infty} \sum_{n=1}^{\infty} L_{i m n} \hat{b}_{i m n}(\tau)+c(\tau)\right]
$$

where

$$
\begin{aligned}
& L_{i m n}=\bar{h} \cos \left(a_{i}\right) \frac{2\left(k_{m}^{2}+k_{n}^{2}-a_{i}^{2}\right)}{\left(2 k_{m} k_{n}\right)^{2}-\left(k_{m}^{2}+k_{n}^{2}-a_{i}^{2}\right)^{2}} \\
& \hat{b}_{i m n}=\int_{0}^{\tau} \mathrm{e}^{-\varsigma_{1} a_{i}^{2}(\tau-\xi)} \bar{\psi}_{n}(\xi) \bar{\psi}_{m}(\xi) \mathrm{d} \xi \\
& c(\tau)=\frac{\sin \left(a_{i}\right)}{4 a_{i}} \frac{4 \omega\left(1-\mathrm{e}^{-\varsigma_{1} a_{i}^{2} \tau}\right)+a_{i}^{4} \varsigma_{1}^{2}(1-\cos (2 \omega \tau))-2 \omega a_{i}^{2} \varsigma_{1} \sin (2 \omega \tau)}{4 \omega^{2} \varsigma_{1} a_{i}^{2}+a_{i}^{6} \varsigma_{1}^{3}}
\end{aligned}
$$

Substituting expression (35) into expression (33), it obtains

$$
\bar{T}(\bar{z}, \tau)=\sum_{i=1}^{\infty} \frac{\bar{h}^{2}+a_{i}^{2}}{\bar{h}+\bar{h}+a_{i}^{2}}\left[\sum_{m=1}^{\infty} \sum_{n=1}^{\infty} L_{i m n} \hat{b}_{i m n}+c(\tau)\right] \cos \left(a_{i} \bar{z}\right)
$$

\subsection{Elastic Field}

After obtaining Lorentz force $\bar{f}_{z}$ and temperature field distribution $\bar{T}, \bar{F}_{z}$ and $N^{T}$ are obtained according to formula (18), and then substitute it in Equation (17). Applying Galerkin method, the initial boundary value problem (17a), (17b) and (21)-(23). Assume that the deflection $\bar{w}(\bar{r}, \tau)$ satisfies with the boundary condition (21) and (22), and has a separable form of time and space as follows

$$
\bar{w}=f(\tau)\left(1-2 \bar{r}^{2}+\bar{r}^{4}\right)
$$


Substituting expression (37) into Equation (17a), the solution of equation (17a) satisfying with boundary Condition (21) and (22) is

$$
\begin{aligned}
\bar{u}(\bar{r}, \tau)= & k f(\tau)\left(\frac{24-16 v}{15} \bar{r}+\frac{4 v-2}{3} \bar{r}^{2}+\frac{1-4 v}{15} \bar{r}^{4}\right) \\
& +f^{2}(\tau)\left[\frac{6-3 v}{6} \bar{r}+(1-3 v) \bar{r}^{3}-\frac{8-2 v}{3} \bar{r}^{5}+\frac{6-v}{6} \bar{r}^{7}\right]
\end{aligned}
$$

Applying Galerkin integral to Equation (17b), it obtains

$$
\begin{aligned}
& \int_{0}^{1} \int_{0}^{2 \pi}\left[\left(\bar{r} \frac{\partial^{3} \bar{w}}{\partial \bar{r}^{3}}+\frac{\partial^{2} \bar{w}}{\partial \bar{r}^{2}}-\frac{1}{\bar{r}} \frac{\partial \bar{w}}{\partial \bar{r}}\right)\right. \\
& -\varsigma_{2} \bar{r}\left\{\left[\frac{\partial \bar{u}}{\partial \bar{r}}+k \frac{\partial \bar{w}}{\partial \bar{r}}+\frac{1}{2}\left(\frac{\partial \bar{w}}{\partial \bar{r}}\right)^{2}+v \frac{\bar{u}}{\bar{r}}\right]-N^{T}\right\}\left(k+\frac{\partial \bar{w}}{\partial \bar{r}}\right) \\
& \left.-\left(\bar{P}_{z}+\bar{F}_{z}\right) \bar{r}^{2}-\varsigma_{3} \int_{0}^{1} \bar{r} \frac{\partial^{2} \bar{w}}{\partial \tau^{2}} \mathrm{~d} \bar{r}\right] \frac{\partial \bar{w}}{\partial f} \bar{r} \mathrm{~d} \theta \bar{r}=0
\end{aligned}
$$

The dynamic response formula expressed by the deflection of the shell can be obtained

$$
a_{1} \frac{\mathrm{d}^{2} f}{\mathrm{~d} \tau^{2}}+a_{2} f^{3}+a_{3} f^{2}+a_{4} f-p_{0}-p_{1}-p_{2}=0
$$

In which

$$
\begin{aligned}
& a_{1}=\varsigma_{3} \int_{0}^{1} \bar{r}\left(\int_{0}^{1} \bar{r} G_{1} \mathrm{~d} \bar{r}\right) G_{1} \mathrm{~d} \bar{r}, \quad a_{2}=\varsigma_{2} \int_{0}^{1}\left[G_{3}^{\prime}+\frac{1}{2} G_{1}^{\prime 2}+\frac{v}{\bar{r}}\right] G_{1}^{\prime} G_{1} \bar{r}^{2} \mathrm{~d} \bar{r}, \\
& a_{3}=\varsigma_{2} \int_{0}^{1} \bar{r}^{2}\left\{k\left[\frac{v}{r} G_{3}+G_{3}^{\prime}+\frac{1}{2} v G_{1}^{\prime 2}\right]+\left[G_{2}^{\prime} G_{1}^{\prime}+k G_{1}^{\prime 2}+\frac{v}{r} G_{2} G_{1}^{\prime}\right]\right\} G_{1} \mathrm{~d} \bar{r}, \\
& a_{4}=\int_{0}^{1}\left\{-\left[\bar{r} G_{1}^{\prime \prime \prime}+G_{1}^{\prime \prime}-\frac{1}{\bar{r}} G_{1}^{\prime}\right]+\varsigma_{2} \bar{r} k\left[G_{4}^{\prime}+k G_{1}^{\prime}+\frac{v}{\bar{r}} G_{2}\right]\right\} G_{1} \bar{r} \mathrm{~d} \bar{r}, \\
& p_{0}=\bar{P} \int_{0}^{1} \bar{r}^{3} G_{1} \mathrm{~d} \bar{r}_{z}, \quad p_{1}=-\varsigma_{2} \bar{N}^{T} \int_{0}^{1}\left(k+G^{\prime}\right) G_{1} \bar{r}^{2} \mathrm{~d} \bar{r}, \quad p_{2}=\bar{F}_{z} \int_{0}^{1} \bar{r}^{3} G_{1} \mathrm{~d} \bar{r}
\end{aligned}
$$

where

$$
\begin{aligned}
& G_{1}(\bar{r})=1-2 \bar{r}^{2}+\bar{r}^{4}, \quad G_{2}(\bar{r})=\frac{24-16 v}{15} \bar{r}+\frac{4 v-2}{3} \bar{r}^{2}+\frac{1-4 v}{15} \bar{r}^{4} \\
& G_{3}(\bar{r})=\frac{6-3 v}{6} \bar{r}+(1-3 v) \bar{r}^{3}-\frac{8-2 v}{3} \bar{r}^{5}+\frac{6-v}{6} \bar{r}^{7}
\end{aligned}
$$

Numerical solution of the dynamic response Equation (40) is obtained by Runge-Kutta method, the response relation of deflection and time is obtained.

\section{Numerical Example}

The shallow conical thin shell is shown in Figure 1, which is made of aluminum and subjected to mechanical load and time-varying magnetic field. Assume that the expression of the function $\phi(\tau)$ of time-varying applied magnetic field is shown as follows.

$$
\phi(\tau)=\sin (\omega \tau)
$$

where $\omega$ is the non-dimensional angular frequency of magnetic field. The 
physical parameters of the shallow conical thin shell are as follows

$$
\begin{gathered}
\mu=4 \pi \times 10^{-7} \mathrm{H} / \mathrm{m}, \quad \sigma=3.42 \times 10^{7} \mathrm{~s} / \mathrm{m}, \quad c=2.7 \times 10^{3} \mathrm{~J} / \mathrm{kg}, \\
\rho=0.9 \times 10^{3} \mathrm{~kg} / \mathrm{m}^{3}, \quad k=92.6 \times 10^{-6} \mathrm{~m}^{2} / \mathrm{s}, \quad v=0.3, \quad E=70 \mathrm{GPa}, \\
\alpha=24 \times 10^{-6} k^{-1}, \quad h=2 \mathrm{~mm}, \quad a=0.2 \mathrm{~m}, \quad \Lambda=2041 / \mathrm{m}
\end{gathered}
$$

According to the above analysis, numerical calculation is obtained by Matlab, the results is shown in Figures 2-7.

Figure 2 and Figure 3 demonstrate the curve of magnetic field $\bar{H}_{r}$ and

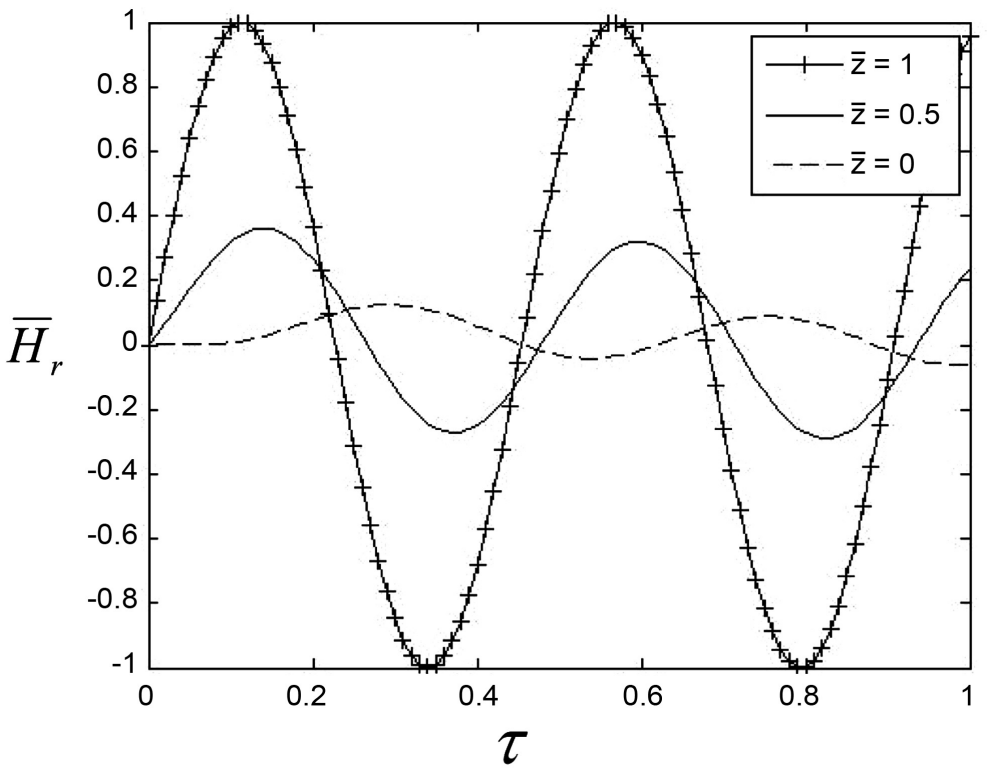

Figure 2. Curve of Magnetic field intensity $\bar{H}_{r}$ vary with time.

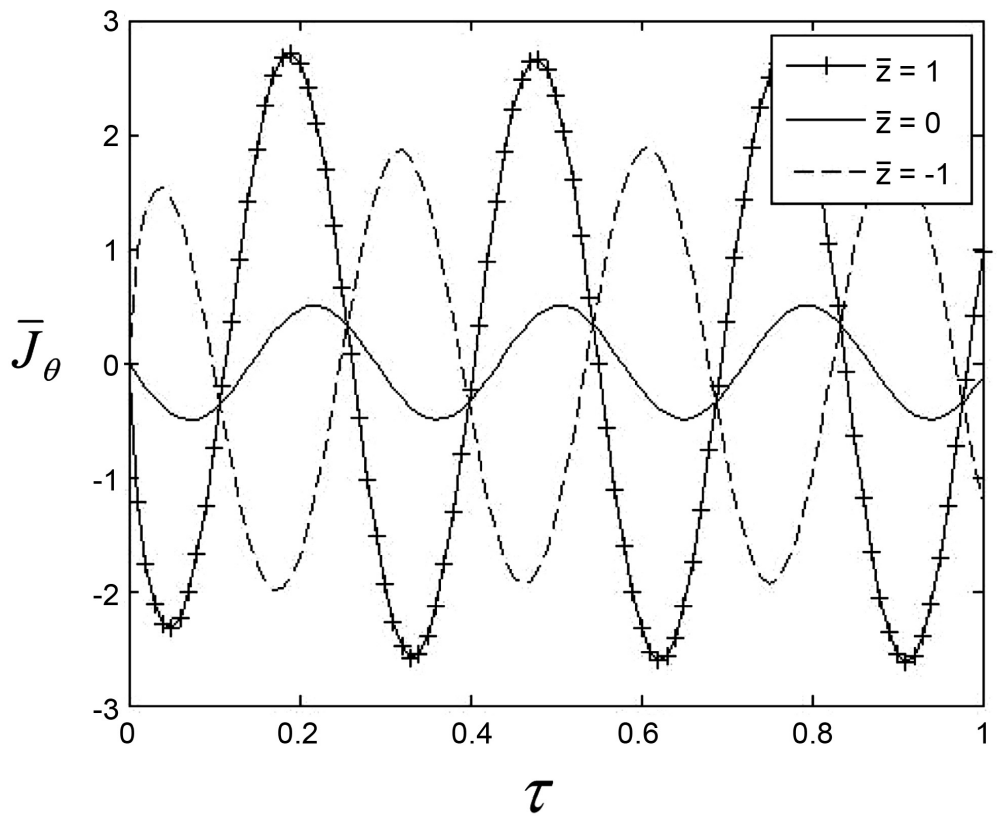

Figure 3. Curve of induced current $\bar{J}_{\theta}$ vary with time. 


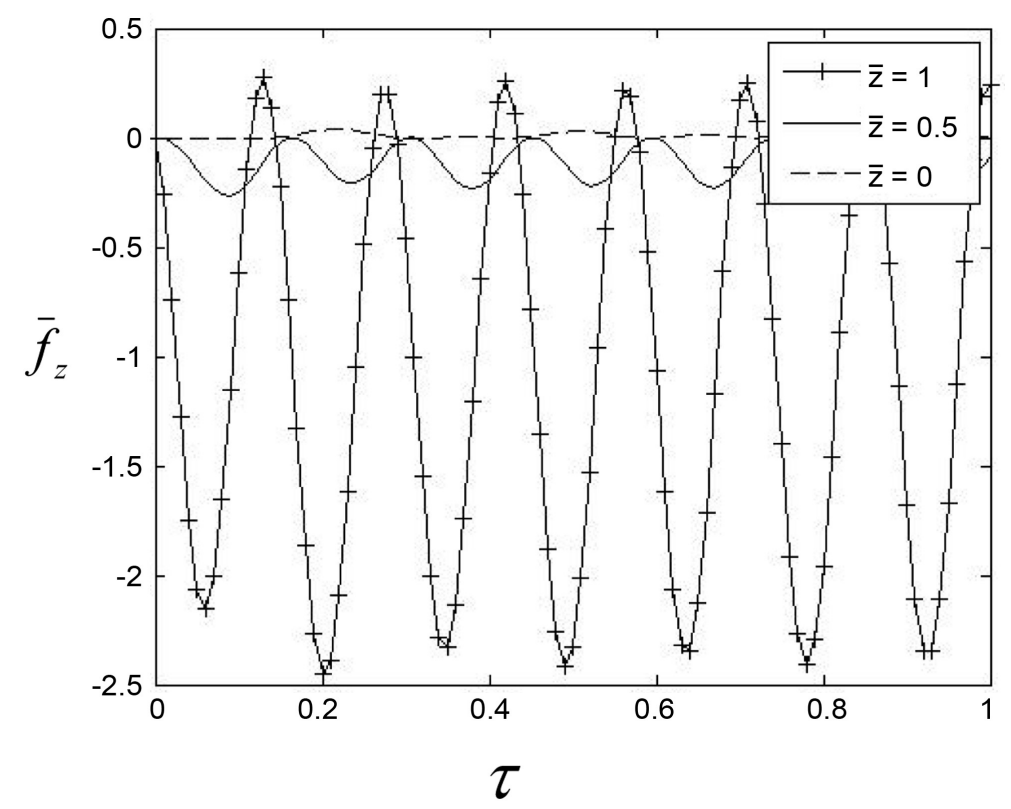

Figure 4. Curve of Lorentz force $\overline{f_{z}}$ vary with time.

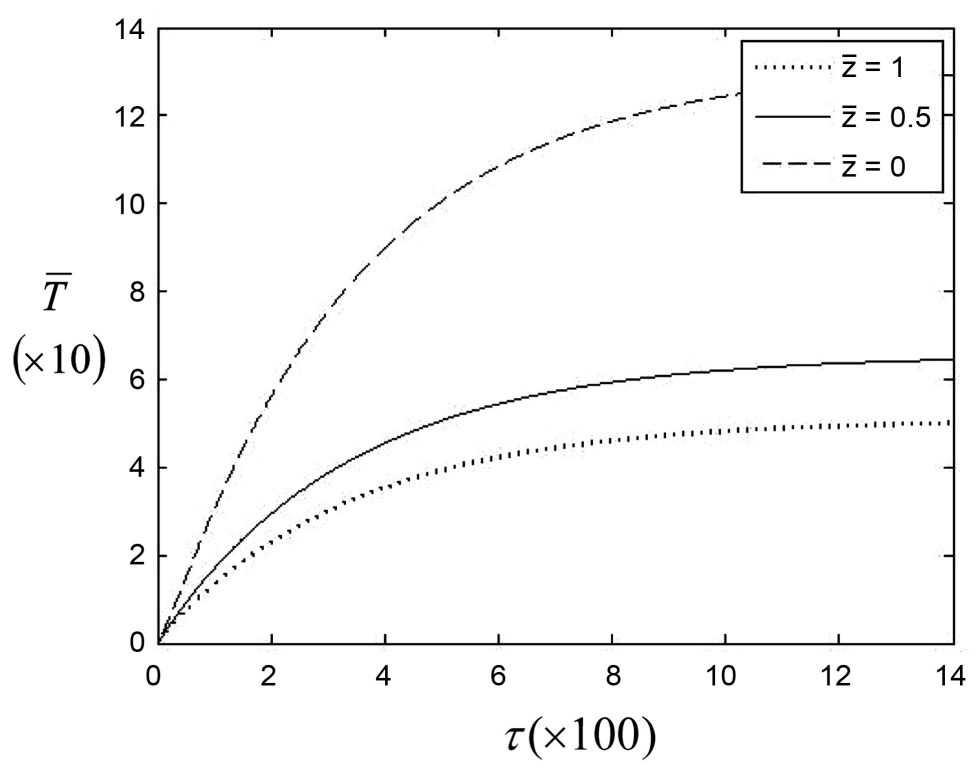

Figure 5. Curve of temperature $\bar{T}$ vary with time.

induced current $\bar{J}_{\theta}$ in different thickness varying with time in the different thickness. It can be seen from Figure 2 that magnetic field $\bar{H}_{r}$ and induced current $\bar{J}_{\theta}$ show sinusoidal variation. The $\bar{H}_{r}$ amplitude gradually increases to 1 when $\mathrm{z}$ is in the interval $[0,1]$ in Figure 2. However, the induced current $\bar{J}_{\theta}$ amplitude deceases first then increases when $z$ is in the interval $[0,1]$ in Figure 3. the outermost surface of shallow conical shell has the maximum induced current amplitude. Figure 4 is the curve of Lorentz force $\bar{f}_{z}$ varying with time in different thickness when $\bar{r}=0.5$, it can be seen that Lorentz force shows sinusoidal variation, and vibration amplitude of shallow conical shell 


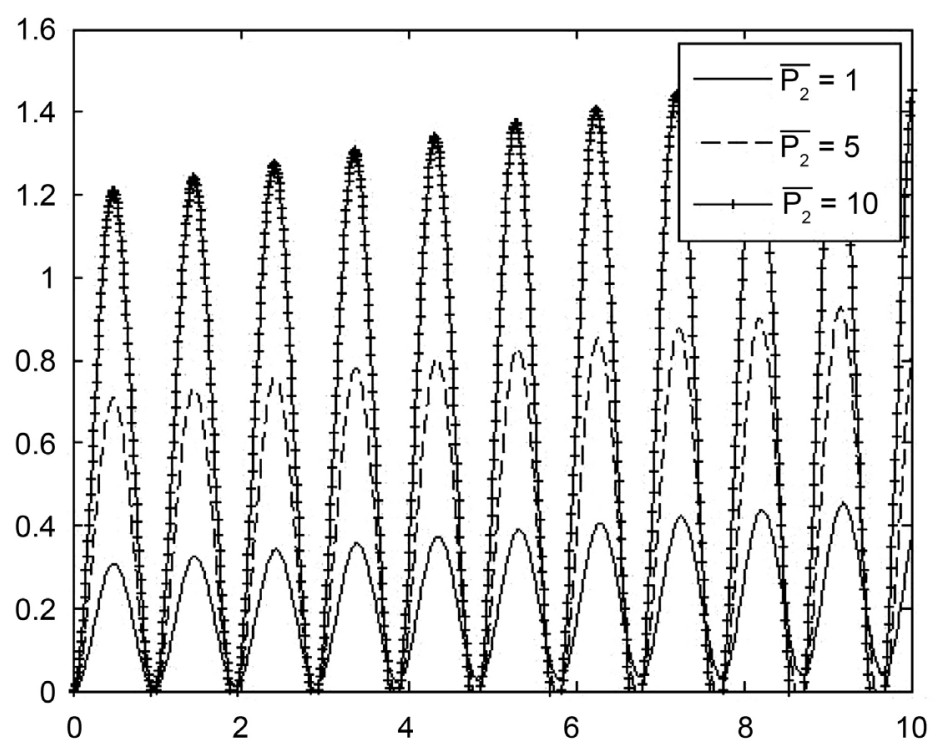

Figure 6. Curve of deflection $\omega$ vary with time in different mechanical load $\left(\bar{r}=0, H_{0}=0.01 / \mu\right)$.

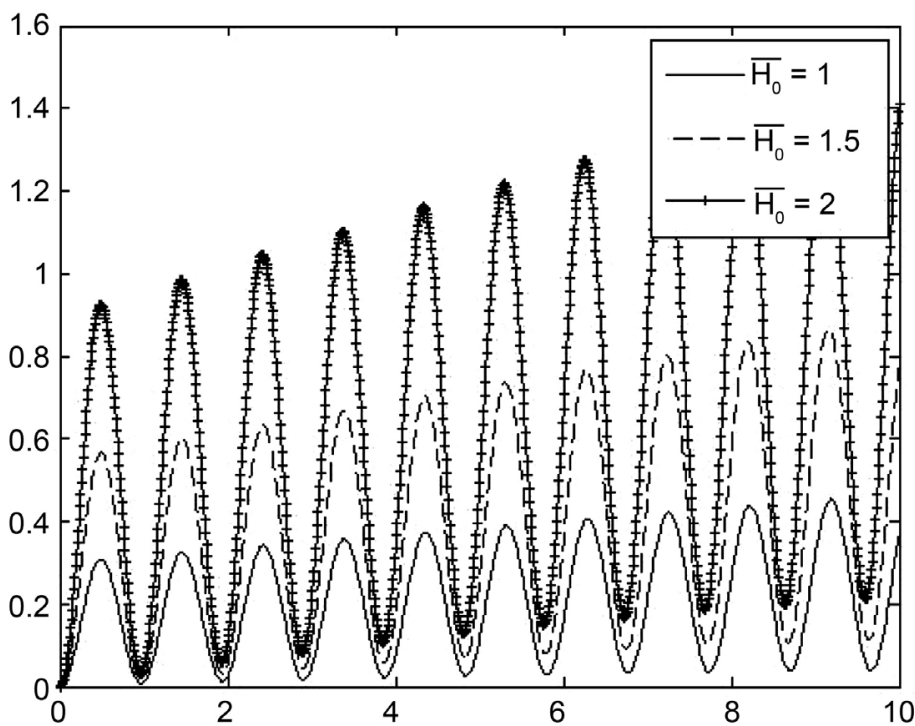

Figure 7. Curve of deflection $\omega$ vary with time in different magnetic field intensity $\left(\bar{r}=0, P_{z}=10^{4} \mathrm{~N}\right)$.

decreases gradually from surface to central face.

Figure 5 is the curve of temperature $\bar{T}$ vary with time in different thickness when $\bar{s}=0.5$, it can be seen that temperature reaches a steady state when the time is long enough,

Figure 6 shows the curve of deflection $\bar{w}$ vary with time in different mechanical load which is expressed by non-dimension mechanical load $\bar{P}_{z}=10^{-3} P_{z}$. It can be seen that mechanical load has an effect on the vibration amplitude of deflection $\bar{w}$, but has no effect on vibration frequency. The vibration amplitude of deflection $\omega$ increases as mechanical load increases. 
Figure 7 shows the curve of deflection $\bar{w}$ vary with time in different applied magnetic field intensity which is expressed by non-dimension magnetic field intensity $\bar{H}_{0}=100 H_{0}$. It can be seen that vibration amplitude increases with the increase of the magnetic field strength.

\section{Conclusions}

Based on Maxwell's equations, heat conduction equation and nonlinear equations of classical plates and shells, the dynamic response study on shallow conical shell's thermo-magneto-elastic behavior in a time-dependent magnetic field is presented. Some conclusions can be obtained through the calculation and analysis of shallow conical shell instances:

1) In the condition that other parameters are constant, mechanical load has an effect on the displacement amplitude of shallow conical shell, but has no effect on the vibration frequency.

2) In the condition that other physical parameters are invariable, the strength of applied magnetic field has influence to the displacement, but has no effect on the vibration frequency.

3) The stress and strain of plate and shell can be controlled when the parameters of magnetic field and mechanical load change appropriately. It has a certain reference value to the practical application of magneto-elastic coupling theory.

\section{Conflicts of Interest}

The authors declare no conflicts of interest regarding the publication of this paper.

\section{References}

[1] Zhou, Y.H. and Zheng, X.J. (1999) Electromagnetic Solid Structural Mechanics. Science Press, Peking.

[2] Bai, X.Z. (2006) Magnetic Base Plate and Shell Elasticity. Science Press, Peking.

[3] Xing, Y.F. and Liu, B. (2010) A Differential Quadrature Analysis of Dynamic and Quasi-Static Magneto-Thermo-Elastic Stresses in a Conducting Rectangular Plate Subjected to an Arbitrary Variation of Magnetic Field. International Journal of Engineering Science, 48, 1944-1960. https://doi.org/10.1016/j.ijengsci.2010.06.010

[4] Higuchi, M., Tanigawa, Y. and Kawamura, R. (2007) Magneto-Thermo-Elastic Stresses Induced by a Transient Magnetic Field in a Conducting Solid Circular Cylinder. Journal of Thermal Stresses, 44, 5316-5335.

[5] Tanigawa, Y., Higuchi, M. and Kawamura, R. (2008) Dynamic and Quasi-Static Behaviors of Magneto-Thermo-Elastic Stresses in a Conducting Hollow Circular Cylinder Subjected to an Arbitrary Variation of Magnetic Field. International Journal of Mechanical Sciences, 50, 365-379. https://doi.org/10.1016/j.ijmecsci.2007.11.001

[6] Tu, J.X., Wang, Z.R., Li, Y.Z. and Wang, P. (2014) Nonlinear Random Vibration of a Current Carrying Circular Plate in a Magnetic Field. Journal of Mechanical Strength, 36, 504-509.

[7] Wang, P., Chen, S.M. and Wang, Z.R. (2013) Bifurcation and Chaos of a Thin Rec- 
tangular Plate Simply Supported with Large Deflection in a Coupled Environment of Heating, Force and Magnetic Field. Journal of Vibration and Shock, 32, 129-134.

[8] Milazzo, A. (2013) A One-Dimensional Model for Dynamic Analysis of Generally Layered Magneto-Electro-Elastic Beams. Journal of Sound and Vibration, 332, 465-483. https://doi.org/10.1016/j.jsv.2012.09.004

[9] Lang, Z. and Li, X. (2013) Buckling and Vibration Analysis of Functionally Graded Magneto-Electro-Thermo-Elastic Circular Cylindrical Shells. Applied Mathematical Modelling, 37, 2279-2292. https://doi.org/10.1016/j.apm.2012.05.023

[10] Kattimani, S.C. and Ray, M.C. (2015) Control of Geometrically Nonlinear Vibrations of Functionally Graded Magneto-Electro-Elastic Plates. International Journal of Mechanical Sciences, 99, 154-167. https://doi.org/10.1016/j.ijmecsci.2015.05.012

\section{Notation Index}

1. a: radius of the shell

2. Thickness of the shell

3. $\varphi$ : the cone Angle

4. $E_{\theta}: \theta$ direction of electric field intensity

5. $B_{r}: r$ direction of magnetic induction intensity

6. $H_{r}: r$ direction of magnetic field intensity

7. J: current density

8. $\sigma$. electrical conductivity

9. $\mu$ : magnetic permeability

10. $\rho$ : material mass density

11. $C$ : the specific heat capacity of the material

12. $K$ : thermal conductivity

13. Q: current loss per unit time per volume

14. f. Lorentzforce

15. $u$ : radial displacement of the shell in neutral

16. $w$. deflection of the shell in neutral

17. E: Elastic Modulus

18. v. Poissonratio

19. $\alpha$ : Coefficient of linear thermal expansion

20. $\sigma_{r}: r$ direction of the stress

21. $\sigma_{\theta}: \theta$ direction of the stress

22. $A$ : Tensile rigidity

23. $D$ : Flexural rigidity 